\title{
Les entrepreneures de la région métropolitaine de Montréal dans la nouvelle économie : impacts de l'immigration et du genre
}

\author{
Sylvie Paré, Ph. D. \\ Kelogue Therasme, Doctorant en études urbaines \\ Université du Québec à Montréal
}

\section{INTRODUCTION}

\section{Pendant la dernière décennie, les femmes entrepreneures à Montréal, et particulièrement celles en provenance des groupes minoritaires, ne présentent pas les mêmes caractéristiques que leurs vis-à-vis masculins.}

La problématique relative à l'entrepreneuriat immigrant et de l'essor de nombre de femmes entrepreneures dans les pays développés a été bien documenté. Nous avons déjà démontré que, pendant la dernière décennie, les femmes entrepreneures à Montréal, et particulièrement celles en provenance des groupes minoritaires, ne présentent pas les mêmes caractéristiques que leurs vis-à-vis masculins. Des résultats semblables ont été obtenus lors d'une étude portant sur cinq groupes ethniques à Toronto et à Vancouver. La recherche mentionnée comparait les groupes de Montréal, de Toronto et de Vancouver étant donné que les trois villes en question ont accueilli une importante population d'immigrants. Cependant, étant donné qu'il y a une grande quantité de nouvelles occasions d'affaires dans une économie faisant l'objet de transformations majeures comme c'est le cas à Montréal, nous pensons qu'il est intéressant d'explorer la place des femmes d'affaires immigrantes dans la nouvelle économie ${ }^{1}$.

Cet article propose d'enrichir les connaissances en explorant le degré de participation des femmes immigrantes entrepreneures d'aujourd'hui à la nouvelle économie? Ce sujet n'est pas que d'intérêt universitaire pour les chercheurs qui se penchent sur la mondialisation des échanges économiques et sur la redéfinition de l'économie de Montréal en termes de technologies nouvelles et d'économie du savoir ${ }^{2}$. En effet, il soulève l'intérêt que peuvent présenter les politiques publiques en ce qui a trait aux besoins et/ou à la pertinence des mesures prévues pour soutenir la participation et l'intégration des femmes à la nouvelle économie.

Cette étude sur la participation des femmes immigrantes à la nouvelle économie se limite à la région métropolitaine de Montréal pour deux raisons principales. Premièrement, la majorité des immigrants s'installent dans la région de Montréal, où se concentre une grande variété de groupes ethnoculturels; certains d'entre eux ayant une histoire d'immigration plus ancienne que d'autres (les communautés italienne et juive, par opposition aux communautés latino-américaines et arabes). Deuxièmement, la structure économique de la région de Montréal a subi de profonds changements depuis quelques décennies, qui ont mené, entre autres, à l'émergence de nouvelles applications dans les technologies de l'information ou internet et de nouveaux domaines de spécialisation économique ${ }^{3}$.

Dans la première partie de cet article, nous débattrons des différents angles théoriques sous lesquels a été abordée la définition des principaux concepts tels que le genre, l'entreprenariat, l'immigration et la nouvelle économie. Dans la deuxième partie, nous exposerons la notion de nouvelle économie. Enfin, les résultats de cette recherche seront présentés à la dernière partie. 


\section{LES FEMMES IMMIGRANTES ENTREPRENEURES DANS LA NOUVELLE ÉCONOMIE}

\section{Peu d'études examinent l'entreprenariat immigrant en tenant compte simultanément du genre et du statut d'immigrant.}

Dans une variété de disciplines, les recherches actuelles tiennent fréquemment compte des antécédents sociaux et de la composition des groupes d'entrepreneurs. Cependant, peu d'études examinent l'entreprenariat immigrant en tenant compte simultanément du genre et du statut d'immigrant. Notre hypothèse générale de travail est que la reconfiguration économique de la région métropolitaine observée depuis quelques décennies est accompagnée de changements dans l'origine sociale des entrepreneurs, tant en termes de genre que d'origine ethnique. Dans cette perspective, l'effort consiste implicitement à examiner à la fois la différenciation et l'inégalité entre les entrepreneurs, engendrées tel qu'attendu - tant par les caractéristiques du genre que par celles du statut d'immigrant.

Cette démarche est semblable à celle des études qui examinent les écarts dans le succès entrepreneurial en fonction des caractéristiques reliées aux antécédents et aux acquis en termes de capital humain, et ce, tant pour le travailleur salarié que pour l'entrepreneur". D'une part, nous formulons l'hypothèse selon laquelle les femmes qui développent certains types d'entreprises se heurtent à des problèmes particuliers. D'autre part, nous vérifions empiriquement la pertinence de deux grandes théories qui dominent dans l'ensemble des ouvrages sur l'entreprenariat ethnique et sur l'intégration des immigrants à la société d'accueil : la théorie de l'assimilation et celle de l'adaptation ethnique. Ces théories ont deux principales variantes : la théorie de l'enclave ethnique et celle du transnationalisme ${ }^{5}$.

L'approche fondée sur la différenciation entre les hommes et les femmes (analyse différenciée selon le sexe, communément appelée ADS) met l'accent sur le fait que les deux groupes ne créent pas le même type d'entreprise. Certains chercheurs $^{6}$ pensent que cela peut en fait expliquer les écarts observés dans le taux de succès respectif de chaque groupe, particulièrement quand nous tenons compte du volume de vente. Cependant, dans le contexte actuel de développement d'une nouvelle économie fondée sur les technologies internet et sur le savoir, nous pourrions bien être surpris de constater la persistance de ces écarts entre les entrepreneurs masculins et féminins. Il ressort que les industries de haute technologie, de construction et de fabrication de technologies internet ont été les secteurs dans lesquels les hommes ont traditionnellement dominé, et dans lesquels le nombre d'employés est significativement plus élevé que dans les autres secteurs. Une autre étude a constaté que «les activités des entreprises de propriété féminine sont concentrées dans des domaines traditionnellement réservés aux femmes, avec des rentrées de fonds inférieures à la moyenne des entreprises concentrées dans des domaines traditionnellement réservés aux hommes ${ }^{7}$ ». Cependant, des travaux plus récents ont montré que les entrepreneurs féminins sont plus fortement concentrés dans les commerces de détail et les services tels la finance, l'assurance, l'immobilier et autres services semblables, secteurs qui se situent parmi ceux dont la croissance est la plus rapide ${ }^{8}$.

\section{Les activités des entreprises de propriété féminine sont concentrées dans des \\ domaines traditionnellement réservés aux femmes, avec des rentrées de fonds \\ inférieures à la moyenne des entreprises concentrées dans des domaines \\ traditionnellement réservés aux hommes.}

Il se pourrait donc que les tendances actuelles favorisent la croissance de secteurs économiques où il $\mathrm{y}$ a concentration de femmes. Cela mènerait peut-être à une situation où la différenciation dans le rendement entre les entreprises détenues par des hommes et les entreprises détenues par des femmes s'estomperait au fil du temps. En comparant les différences individuelles entre les femmes entrepreneures qui travaillent dans des secteurs traditionnels et non-traditionnels de l'économie, il ressort que les femmes travaillant 
dans des secteurs à dominante masculine avaient plus de difficultés parce que la création et le développement de telles entreprises exigeaient une planification beaucoup plus serrée, et parce que l'obtention de financement et de crédit présentait de grandes difficultés ${ }^{7}$.

Il semblerait alors que la question de la place des femmes entrepreneures dans la nouvelle économie, leurs conditions d'accès au statut d'entrepreneur et leur succès relatif mérite d'être clarifiés. Cette question reste d'une pertinence d'autant plus grande s'il s'agit de femmes immigrantes ou de femmes faisant partie de groupes minoritaires.

Quand nous examinons cette question sous l'angle du statut d'immigrant ou de l'origine ethnique ou de ces variables combinées, la mise sur pied d'une entreprise peut être tributaire de la taille du groupe d'immigrants dans l'ensemble de l'économie locale. Le type d'entreprises créées par des immigrants peut être plus ou moins semblable à celui des entreprises que l'on retrouve dans l'ensemble de l'économie locale. Alors qu'il y a une grande quantité d'ouvrages sur le thème de l'intégration des immigrants et des groupes minoritaires, il y en a peu sur l'entreprenariat immigrant comme un indicateur d'intégration économique.

\section{L'entrepreneur immigrant se centre sur les activités dont il tire les avantages relatifs que lui confère son appartenance à un groupe ethnique spécifique.}

En général, la recherche sur l'intégration économique et sociale des immigrants à la société d'accueil a été marquée par deux approches importantes. D'abord, la théorie de l'assimilation postule que les valeurs culturelles et les origines immigrantes sont des facteurs qui influencent le développement de l'entreprenariat immigrant ${ }^{5}$. Dans un contexte d'isolement géographique et de ségrégation, l'entrepreneur émergent saisit les occasions d'affaires pour desservir les enclaves ethniques, c'est-à-dire les communautés dont les valeurs et les besoins sont relativement semblables. Pour certains chercheurs, l'absence d'assimilation structurelle et culturelle des entrepreneurs immigrants fait la différence entre le type d'entreprise que ces derniers mettent sur pied et le type d'entreprises créées par des entrepreneurs nés dans le pays. Dans ce cas, l'entrepreneur immigrant tente de satisfaire les besoins de son groupe ethnique, alors que les entrepreneurs nés dans le pays tentent de satisfaire les besoins de la société en général. L'entrepreneur immigrant se centre sur les activités dont il tire les avantages relatifs que lui confère son appartenance à un groupe ethnique spécifique. En même temps, l'immigrant entrepreneur peut s'approprier les valeurs et normes de la société d'accueil en général, ce qui peut l'amener à étendre ses activités en dehors du groupe ethnique en adoptant une démarche qui ressemble à celle des entrepreneurs nés dans le pays. Cependant, ni le niveau de scolarité, ni une formation traditionnelle dans le domaine des affaires, ni la connaissance de la langue dominante ne semblent reliés au succès des entrepreneurs immigrants qui s'insèrent dans l'économie d'ensemble.

Ensuite, la théorie de l'ethno-résilience met l'accent sur deux aspects distincts de l'entreprenariat ethnique. Pour le premier, la communauté ethnique locale offre des possibilités d'affaires beaucoup plus accessibles que dans un marché plus largement ouvert à tous. Le deuxième aspect met l'accent sur les possibilités d'affaires reliées au pays d'origine. Les immigrants entrepreneurs sont considérés comme des entrepreneurs transnationaux, qui «achètent et vendent à l'étranger, voyagent par affaire sur le plan international, investissent dans des entreprises et embauchent même du personnel dans leur pays d'origine $^{5}$ ». Ces entrepreneurs transnationaux réalisent des gains et revenus substantiels de l'ordre de 4000 \$ par mois, un revenu supérieur à celui des entrepreneurs nationaux.

Les immigrants entrepreneurs sont considérés comme des entrepreneurs transnationaux, qui « achètent et vendent à l'étranger, voyagent par affaire sur le plan international, investissent dans des entreprises et embauchent même du personnel dans leurs pays d'origine. 
Le développement de commerces dans des enclaves ethniques ou de commerces d'échanges avec le pays d'origine ne semble pas voulu mais constitue plutôt une sorte de réaction logique aux obstacles et/ou à la discrimination auxquels sont confrontés les immigrants dans le développement de leur projet d'entreprise ${ }^{8}$.

Cette tendance caractérise aussi le développement des entreprises de la nouvelle économie. À partir d'une démarche qualitative et d'entrevues en profondeur, Leung ${ }^{9}$ examine comment le contexte socio-économique du pays d'accueil interagit avec celui du pays d'origine en ce qui concerne le lieu, les pratiques de fonctionnement, les stratégies de marketing et le choix du type d'entreprise à développer. Dans le cas des immigrants entrepreneurs chinois, sa recherche montre clairement l'importance des réseaux ethniques transnationaux dans le développement et la spécialisation des entreprises ethniques. Étant donné la nature qualitative de la recherche de Leung, il n'est pas du tout clair que ses résultats soient applicables à tous les types d'entreprises de la nouvelle économie. Pour mieux saisir l'importance des réseaux ethniques transnationaux, il est important d'examiner le concept de «nouvelle économie ».

\subsection{Définir la nouvelle économie}

Pour définir la nouvelle économie, les recherches recensées présentent trois approches conceptuelles différentes. La première renvoie aux changements structurels et à la révolution technologique qui ont eu lieu depuis les années 1970. Ces changements ont modifié de façon significative et permanente la structure économique des sociétés d'affaires dans une grande variété de secteurs d'activités économiques. Les ressources naturelles et le capital physique sont devenus, de manière significative, moins importants dans la structure de productivité ellemême, tandis que les technologies de l'information ont de plus en plus pénétré tous les types d'activités économiques ${ }^{9}$. En ce qui concerne la Province de Québec, l'attention est centrée sur l'adoption de nouvelles technologies de production par des grandes sociétés commerciales et des petites et moyennes entreprises (PME). En 1989, 90 \% des PME manufacturières au Québec avaient informatisé en partie leurs activités.
Cependant, l'implantation de technologies de l'information en ce qui concerne à la fois l'étendue et le type d'utilisation varie d'une industrie à l'autre ${ }^{10}$.

Une deuxième approche de définition de la nouvelle économie relie les nouvelles technologies de l'information à des secteurs particuliers de l'activité économique ${ }^{11}$. La complexité des nouveaux produits et services est en général limitée à quelques secteurs d'activité, caractérisés par un plus haut degré de connaissances techniques et/ou d'usage de technologies de l'information. Pour cette raison, beaucoup de recherches sur la nouvelle économie se sont limitées aux secteurs d'activités fondés sur les connaissances en économie et sur la production des technologies de l'information, particulièrement aux industries manufacturières, aux projets scientifiques et à certains services professionnels. Ainsi, l'économie des régions métropolitaines est largement caractérisée par la production, la distribution et l'utilisation du savoir relié aux technologies de l'information. En fait, des auteurs utilisent les expressions «nouvelle économie » et « économie du savoir » de façon interchangeable. Néanmoins, en dépit de sa popularité dans la littérature, cette approche élimine les sociétés d'affaires issues de la révolution informatique et passe sous silence le grand progrès réalisé dans les entreprises plus traditionnelles, lesquelles ont intégré les technologies de l'information dans une grande variété de domaines de leurs activités économiques.

La troisième approche de définition de la nouvelle économie met l'accent sur l'introduction de pratiques de gestion renouvelées. En d'autres mots, les nouvelles technologies ne sont pas implantées que dans le processus de production; elles le sont également dans toutes sortes d'aspects des activités de l'entreprise. Pendant les années 1990, les modes de travail des entreprises ont notablement changé par rapport aux années antérieures, tant dans la manière d'organiser que de faire le travail. Conséquemment, les changements suscités par l'utilisation des technologies de l'information ont exigé le recours à de nouveaux modèles et à de nouvelles stratégies de gestion. Si l'utilisation des technologies internet a rendu les processus 
de production et de distribution plus rapides et moins coûteux, elle a également changé la nature de la compétition entre entreprises. Quel que soit le secteur d'activité économique, nous trouvons ainsi des entreprises qui utilisent toujours de vieilles technologies en matière de méthodes de fabrication et de contrôle de la qualité, et celles qui ont introduit des méthodes de production et de contrôle fondées sur les technologies de l'information. Ces dernières sont souvent caractérisées par la présence de programmes de contrôle de la qualité, d'unités de RechercheDéveloppement, de sites Web interactifs avec lesquels le producteur peut échanger directement de l'information avec des sous-traitants et des clients $^{12}$.

L'Internet est l'incubateur de la nouvelle économie à cause de son potentiel de réseautage. $\mathrm{La}$ technologie Internet offre aux gens d'affaires des avantages comparatifs, plus particulièrement en termes de différenciation de produits et services, d'accroissement des occasions d'affaires et de gains de productivité. Cependant, afin d'optimiser les gains que les technologies d'Internet pourraient engendrer, les entreprises doivent transformer leur structure organisationnelle et développer leur capacité à manier les outils technologiques appropriés et à le faire de façon exhaustive. En ce sens, les technologies d'Internet jouent un rôle important dans la revitalisation d'entreprises plus anciennes.

En s'inspirant des approches examinées cidessus et à la lumière d'une synthèse de la littérature, il est possible de définir le concept de nouvelle économie de façon plus large et plus appropriée. Sur ce point, il s'agit du domaine des activités économiques qui sont liées aux nouvelles technologies de l'information et des communications, activités qui intègrent les mises au point technologiques les plus récentes et qui reposent sur un niveau élevé de connaissances. Cette définition renvoie à la capacité d'innovation des entreprises de créer, d'utiliser ou de transformer les technologies de l'information fondées sur le savoir dans une variété de domaines de l'activité économique.

La nouvelle économie est ainsi le terrain fertile d'entreprises créatives, d'entreprises revitalisées et d'entreprises dérivées ${ }^{13}$, tel qu'illustré à la figure 1 .

\section{Figure 1 : Typologie des entreprises de la nouvelle économie}

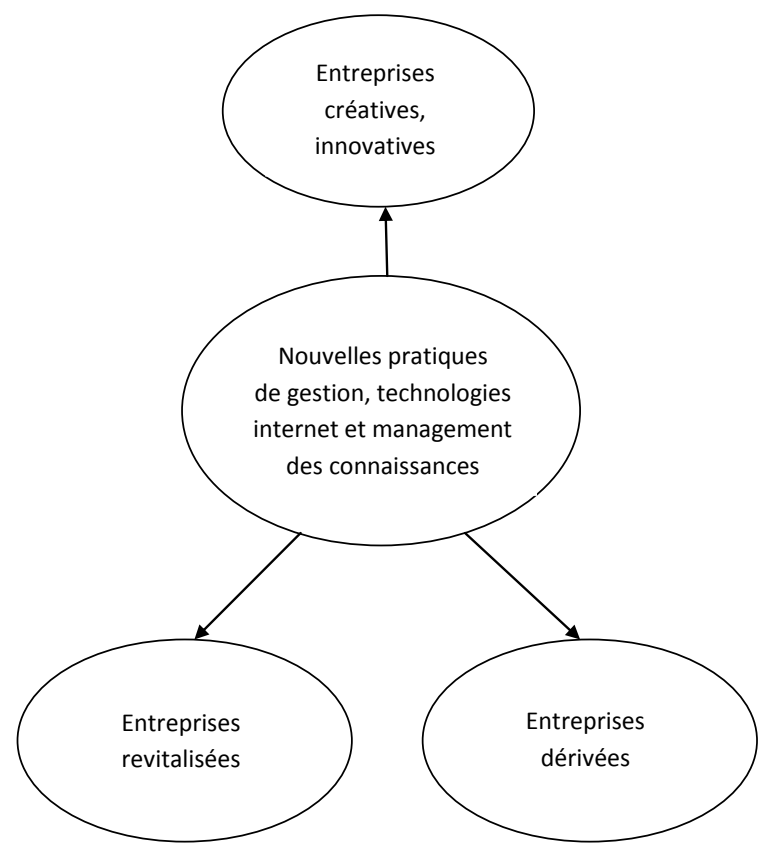


D'abord, les « entreprises créatives » sont celles où sont générées de nouvelles idées, de nouveaux produits et services. Elles participent à la production de connaissances, à la conception de technologies de l'information, et à de nouveaux modèles ou de nouvelles stratégies de gestion (entreprises actives dans le domaine de la fabrication de technologies de l'information ou dans la conception de services reliés à ces technologies et entreprises spécialisées dans la production de connaissances). Ensuite, les «entreprises revitalisées » sont celles, plus traditionnelles, qui ont continué à suivre l'évolution de l'économie du savoir en intégrant des technologies de l'information et en adoptant des modèles et stratégies de gestion nouveaux et plus opportuns. Enfin, les «entreprises dérivées » sont celles qui ont été constituées pour répondre à un besoin de distribution, de marketing ou d'utilisation de produits et services développés par les entreprises créatives.

Malgré la typologie des entreprises de la nouvelle économie, la distribution des entrepreneurs masculins et féminins ne tient pas compte du type d'entreprise fondée. Comme nous l'avons vu précédemment, la nouvelle économie ne se résume pas à quelques secteurs de l'activité économique. Elle s'incarne plus précisément dans une culture de procédés et méthodes de gestion de plus en plus intensément ancrés dans les technologies de l'information et développés dans un contexte de mondialisation de l'activité économique. Il est donc approprié d'examiner l'entrepreneurial féminin en lien avec les technologies de l'information.

\subsection{Entrepreneurs au féminin et technologies de l'information}

La Banque canadienne de développement estime que les femmes dirigent un nombre croissant de petites entreprises issues de secteurs non traditionnels dont la

biotechnologie, la robotique et la fabrication.

Selon diverses recherches antérieures, le nombre des entrepreneurs féminins a considérablement augmenté et pourrait représenter plus de $30 \%$ du nombre total d'entrepreneurs. La Banque canadienne de développement estime que les femmes dirigent un nombre croissant de petites entreprises issues de secteurs non traditionnels dont la biotechnologie, la robotique et la fabrication ${ }^{14}$. Cependant, il se peut que la situation ne soit pas tout à fait la même à Montréal, où la participation des femmes entrepreneures est particulièrement faible, une particularité qui est en partie justifiée par l'intersection de divers marqueurs de l'identité personnelle. À l'exclusion du fait que les femmes entrepreneures sont généralement absentes des grandes entreprises, le nombre de femmes entrepreneures est tributaire de l'intersection de certaines caractéristiques personnelles ${ }^{15}$. Par exemple, des différences significatives ressortent quand nous tenons compte des membres des groupes majoritaires (Canadiens anglais et Canadiens français à Montréal) ou des membres de groupes minoritaires. Les difficultés éprouvées par les entrepreneurs appartenant à des groupes minoritaires peuvent être liées à un faible niveau de scolarité, à leurs carences en termes de compétences pertinentes et à leur exclusion des réseaux informels d'affaires des groupes majoritaires. D'autres travaux portent à croire que, même si les hommes et les femmes ont des résultats scolaires semblables, des différences subsistent entre eux sur le plan des domaines d'études. Sur ce point, plus d'hommes que de femmes se spécialisent dans des branches techniques. Les femmes sont plus susceptibles de compléter leur formation en santé, en commerce, en administration ou en économie, laissant à penser qu'elles ne développeront vraisemblablement pas des entreprises de haute technologie et qu'elles seront moins sensibilisées - ou qu'elles seront fermées à l'adoption de technologies de l'information ${ }^{16}$.

Parmi les recherches publiées sur la nouvelle économie, peu d'entre elles sont centrées sur la présence des femmes dans des entreprises créatives, dérivées ou revitalisées. De plus, il y a moins d'études sur des femmes d'origine immigrante ou faisant partie d'un groupe minoritaire et encore moins sur des femmes appartenant à des minorités « racialisées ». C'est pour relever ce défi que cette recherche se penche sur la place des femmes entrepreneures dans la nouvelle économie, en fonction de leur lieu de naissance, du type d'entreprise qu'elles dirigent et de la pénétration des technologies d'Internet dans leurs organisations. 


\section{MÉTHODOLOGIE}

Les données sont tirées des Répertoires des entreprises Scott, une banque de données semblable au Harris Index aux États-Unis. Ces données répertorient la présence des femmes gestionnaires et le poste qu'elles occupent dans chaque entreprise. De plus, ces répertoires fournissent des données sur le type d'entreprise (selon le système de classification des industries de l'Amérique du Nord), les produits et services offerts, la présence de personnel affecté à la recherche et au développement, l'existence d'un site Internet, l'utilisation des technologies de messagerie électronique comme moyen de communication, le lieu de l'entreprise, sa taille, le nombre d'employés, l'année de sa constitution en société et le volume de ses ventes.

Il faut toutefois souligner que ce répertoire comporte des lacunes. Il est incomplet car les entreprises nouvellement créées ne se sont pas enregistrées avant l'année suivante. Aussi, les entrepreneurs appartenant à des groupes minoritaires ne savent pas qu'ils doivent être enre- gistrés dans cette banque de données. Néanmoins, le Scott reste un outil précieux pour étudier les caractéristiques tant des entrepreneurs que des entreprises. Dans une précédente recherche fondée sur les données des répertoires Scott, nous avons trouvé une vaste répartition géographique d'entreprises créatives dans l'économie du savoir ou relevant des technologies de l'information. Au même moment, la plupart de ces entreprises cherchaient à se loger dans le centre-ville de la région métropolitaine de Montréal ${ }^{1}$.

À partir des répertoires Scott 2007, nous avons ainsi recensé 22872 entreprises situées dans la région de Montréal. En nous servant des codes du système de classification des industries de l'Amérique du Nord, nous avons dégagé un sous-ensemble de 2789 entreprises (12\%), qui peuvent être caractérisées comme relevant de 71 secteurs différents d'activités en technologies de l'information, ou comme des activités fondées sur le savoir (voir tableau 1).

Tableau 1 : Type d'entreprises de la nouvelle économie (2006)

\begin{tabular}{|l|l|l|}
\hline Type d'entreprises & Nombre & Pourcentage \\
\hline Fabrication TI & 282 & 10,1 \\
\hline Services TI & 999 & 35,8 \\
\hline Fondées sur le savoir & 1508 & 54,1 \\
\hline \hline Ensemble & 2789 & 100,0 \\
\hline
\end{tabular}

Source : Les répertoires d'entreprises Scott, 2007

Les répertoires Scott fournissent une grande quantité de renseignements concernant la propriété et/ou la direction de chaque entreprise, comprenant le nom de famille, le genre des propriétaires, la présence de copropriétaires et d'autres personnels de gestion. La personne qui occupe un poste de responsabilité est celle qui est au centre du pouvoir décisionnel dans une entreprise donnée. Elle représente l'indicateur le plus approprié pour les fins d'analyse. Quand le propriétaire de l'entreprise est sur la liste du répertoire Scott, cette personne est toujours mentionnée comme «la personne qui occupe un poste de responsabilité $»$. Pour ces raisons, la «personne qui occupe un poste de responsabilité » est la variable que nous avons retenue pour notre étude. Selon les données du tableau 2, plus de $85 \%$ des entreprises ont un propriétaire ou un associé directeur (ou un associé directeur général) qui occupe une fonction de responsabilité en tant que président, propriétaire ou gestionnaire partenaire, c'est-àdire un poste qui peut être considéré comme entrepreneurial. 
Tableau 2 : Nombre de postes de responsabilité occupés dans les entreprises de Montréal (2006)

\begin{tabular}{|l|l|l|}
\hline Poste & Nombre & Pourcentage \\
\hline \hline Président/Coprésident & 10979 & 49,6 \\
\hline Propriétaire/Copropriétaire & 4348 & 19,6 \\
\hline Associé & 3545 & 16,0 \\
\hline Partenaire & 692 & 3,1 \\
\hline Vice-président & 418 & 1,9 \\
\hline Président du conseil & 386 & 1,7 \\
\hline Autres titres & 1789 & 8,1 \\
\hline Ensemble & 22157 & 100,0 \\
\hline
\end{tabular}

Source : Les répertoires d'entreprises Scott, 2007

\section{RÉSULTATS ET DISCUSSIONS}

\subsection{Entrepreneuriat immigrant dans la nouvelle économie}

En plus de l'indicateur relatif au poste de responsabilité occupé dans l'entreprise, nous avons examiné la présence de femmes dans des postes de gestionnaire. Bien qu'il n'y ait que $16,7 \%$ de femmes qui occupent un poste de responsabilité dans les entreprises de la région métropolitaine de Montréal, il y a toutefois $34,2 \%$ de ces entreprises qui signalent la présence d'au moins une femme occupant un poste de gestionnaire.

L'identification des entrepreneurs immigrants est plus complexe puisque le seul indice que fournissent les répertoires Scott est le nom de famille des personnes qui ont été identifiées comme celles occupant un poste de responsabilité. En général, la société québécoise reste pour une grande part marquée par un clivage entre deux groupes ethniques dominants: les Canadiens français et les Canadiens anglais. Étant donné que la plupart des immigrants sont d'arrivée récente, les noms de famille qui ont une origine ni française ni anglaise/écossaise/irlandaise identifient vraisemblablement les immigrants de date récente, leurs enfants ou leurs petits-enfants ${ }^{1}$. Bien que cette méthode ne permette pas d'identifier les personnes d'origine immigrante de façon tout à fait sûre, son utilisation pour la population du Québec a été une expérience réussie ${ }^{17}$. À l'aide de dictionnaires généalogiques, nous avons réussi à diviser l'ensemble des entreprises en trois groupes : $1^{\circ}$ origine anglaise; $2^{\circ}$ origine française et $3^{\circ}$ autres origines. Le tableau 3 montre que près du tiers de toutes les personnes occupant un poste de responsabilité dans la région de Montréal provient de groupes autres que des deux groupes majoritaires.

Tableau 3 : Origine du nom de famille des personnes en responsabilité dans les entreprises de Montréal (2006)

\begin{tabular}{|l|l|l|}
\hline $\begin{array}{l}\text { Origine du nom de } \\
\text { famille }\end{array}$ & Nombre & Pourcentage \\
\hline \hline Française & 12452 & 56,2 \\
\hline Anglaise & 2461 & 11,1 \\
\hline Autre & 7238 & 32,7 \\
\hline Ensemble & 22151 & 100,0 \\
\hline
\end{tabular}

Source : Les répertoires d'entreprises Scott, 2007 


\section{Il en ressort aussi que le groupe d'origine immigrante qui mène des activités entrepreneuriales est relativement plus important que les immigrants dans la population totale du Québec.}

Il en ressort aussi que le groupe d'origine immigrante qui mène des activités entrepreneuriales est relativement plus important que les immigrants dans la population totale du Québec. Bien qu'elle ne corresponde pas complètement à notre définition de ce qu'est un groupe d'origine immigrante, la proportion de la population dont la langue maternelle est autre que le français ou l'anglais n'était que de $21,8 \%$; ceux qui ont comme langue maternelle le français représentent $65,7 \%$ de la population totale, un pourcentage qui est lié de près au concept d'origine française au Québec. Ainsi, en termes d'activités entrepreneuriales, les personnes d'origine française semblent être sous-représentées. La catégorie «autres origines» est surreprésentée. Il s'agit là d'un indicateur de la contribution des entrepreneurs immigrants à l'ensemble de l'activité économique.

\subsection{La relation entre genre et type d'entreprise}

En plus du système de classification décrivant les entreprises de la nouvelle économie, nous avons aussi fait la différence entre les entreprises traditionnelles qui ont progressé grâce à l'adoption et à l'intégration des technologies de l'Internet et celles qui ne l'ont pas fait. Les entreprises qui se caractérisent par la présence d'un site Web et qui utilisent le courriel, et/ou qui ont à leur service un spécialiste des technologies internet ont été, par le fait même, classées «entreprises traditionnelles avec technologies internet ». Celles qui n'ont visiblement pas fait d'effort pour atteindre le plus vaste marché transnational utilisant des technologies Web ont été classées «entreprises traditionnelles sans technologies internet ». Le tableau 4 présente la distribution selon le type d'entreprises (présence de TI ou non) et le genre de la personne en responsabilité.

Tableau 4 : Type d'entreprises selon le genre à Montréal (2006)

\begin{tabular}{|l|l|l|l|}
\hline Type d'entreprises & $\begin{array}{l}\text { Dirigée par } \\
\text { un homme }\end{array}$ & $\begin{array}{l}\text { Dirigée par } \\
\text { une femme }\end{array}$ & Ensemble \\
\hline \hline Nouvelle économie & $13,4 \%$ & $7,5 \%$ & $12,4 \%$ \\
\hline Traditionnelle avec TI & $61,3 \%$ & $69,0 \%$ & $62,6 \%$ \\
\hline Traditionnelle sans TI & $25,3 \%$ & $23,5 \%$ & $25,0 \%$ \\
\hline Ensemble & $100,0 \%$ & $100,0 \%$ & $100,0 \%$ \\
\hline$(\mathrm{N})$ & 18443 & 3692 & 21135 \\
\hline
\end{tabular}

Source : Les répertoires d'entreprises Scott, 2007

Les femmes sont significativement plus susceptibles d'être sous-représentées dans la nouvelle économie. Cependant, elles dirigent plus d'entreprises traditionnelles utilisant des technologies internet que leurs équivalents masculins.

À la lumière du tableau 4, les femmes sont significativement plus susceptibles d'être sousreprésentées dans la nouvelle économie.
Cependant, elles dirigent plus d'entreprises traditionnelles utilisant des technologies internet que leurs équivalents masculins. Ce résultat coïncide exactement avec les résultats de travaux antérieurs ${ }^{1}$, lesquels ont démontré que les entrepreneurs féminins doivent franchir plus d'obstacles que les hommes pour obtenir des fonds de démarrage.

Cette sous-représentation n'est qu'une des manifestations d'une tendance plus accentuée selon laquelle les femmes de certains groupes 
sont plus susceptibles que les autres de réussir à pénétrer le marché à titre d'entrepreneur. Le tableau 5 montre que, dans l'économie montréalaise prise dans son ensemble, les femmes d'origine française sont présentes dans des proportions significativement plus élevées que celles des deux autres groupes. Les femmes du groupe anglais et celle d'origine immigrante ont plus de difficultés à concrétiser leur aspiration à devenir entrepreneures.

Les femmes du groupe anglais et celle d'origine immigrante ont plus de difficultés à concrétiser leur aspiration à devenir entrepreneures.

Tableau 5 : Présence des femmes entrepreneures selon le groupe d'origine dans les entreprises à Montréal en 2006

\begin{tabular}{|l|l|l|l|l|}
\hline Genre & Français & Anglais & Autres & Ensemble \\
\hline Masculin & $81,7 \%$ & $86,8 \%$ & $84,9 \%$ & $83,3 \%$ \\
\hline Féminin & $18,3 \%$ & $13,2 \%$ & $15,1 \%$ & $16,7 \%$ \\
\hline Ensemble & $100,0 \%$ & $100,0 \%$ & $100,0 \%$ & $100,0 \%$ \\
\hline (N) & 12445 & 2455 & 7235 & 22135 \\
\hline
\end{tabular}

Source : Les répertoires d'entreprises Scott, 2007

Les affinités entrepreneuriales des femmes, leurs champs de compétences et leur talent à saisir les occasions d'affaires dans la nouvelle économie sont semblables à ceux de leurs équivalents masculins.

Cependant, une fois que des entreprises de la nouvelle économie ont été créées par des femmes, les données montrent qu'il n'y a pas de différence notable entre le type d'entreprises qu'elles dirigent et le type de celles dirigées par des hommes. À la lumière du tableau 6 , les affinités entrepreneuriales des femmes, leurs champs de compétences et leur talent à saisir les occasions d'affaires dans la nouvelle économie sont semblables à ceux de leurs équivalents masculins.

Tableau 6 : Type d'entreprises de la nouvelle économie selon le genre, Montréal (2006)

\begin{tabular}{|l|l|l|l|}
\hline Type d'entreprises & $\begin{array}{l}\text { Dirigée par un } \\
\text { homme }\end{array}$ & $\begin{array}{l}\text { Dirigée par } \\
\text { une femme }\end{array}$ & Ensemble \\
\hline \hline TI Manufacturière & $10,4 \%$ & $9,0 \%$ & $10,3 \%$ \\
\hline TI Services & $35,5 \%$ & $36,0 \%$ & $35,6 \%$ \\
\hline Fondée sur le savoir & $54,1 \%$ & $55,0 \%$ & $54,2 \%$ \\
\hline Ensemble & $100,0 \%$ & $100,0 \%$ & $100,0 \%$ \\
\hline$(\mathrm{N})$ & 2474 & 278 & 2752 \\
\hline So & &
\end{tabular}

Source : Les répertoires d'entreprises Scott, 2007 
3.3 La relation entre l'origine immigrante et le type de commerce

Le tableau 7 présente la distribution des entreprises de femmes, selon l'appartenance aux groupes français et anglais et au groupe autres (d'origine immigrante). Les différences observées ne sont pas statistiquement significatives. Ni l'appartenance au statut de majoritaire, ni l'appartenance au statut de minoritaire, ni les origines, qu'elles soient anglaises, françaises ou autres n'ont un impact sur le type d'entreprises dirigées par des femmes.

Ni l'appartenance au statut de majoritaire, ni l'appartenance au statut de minoritaire, ni les origines, qu'elles soient anglaises, françaises ou autres n'ont un impact sur le type d'entreprises dirigées par des femmes.

Tableau 7 : Type d'entreprises dirigées par des femmes, selon l'origine immigrante à Montréal (2006)

\begin{tabular}{|l|l|l|l|l|}
\hline Type d'entreprises & Français & Anglais & Autres & Ensemble \\
\hline Nouvelle économie & $7,0 \%$ & $9,3 \%$ & $8,0 \%$ & $7,5 \%$ \\
\hline Traditionnelle avec TI & $69,6 \%$ & $68,8 \%$ & $67,7 \%$ & $69,0 \%$ \\
\hline Traditionnelle sans TI & $23,4 \%$ & $21,9 \%$ & $24,3 \%$ & $23,5 \%$ \\
\hline Ensemble & $100,0 \%$ & $100,0 \%$ & $100,0 \%$ & $100,0 \%$ \\
\hline (N) & 2278 & 324 & 1090 & 3692 \\
\hline
\end{tabular}

Source : Les répertoires d'entreprises Scott, 2007

Les femmes du groupe majoritaire français sont plus susceptibles de créer des entreprises fondées sur le savoir que les femmes des deux autres groupes, lesquels sont plus fortement concentrées dans les entreprises de services avec $\mathbf{T I}$.

Pour vérifier si le statut d'immigrant peut avoir un impact sur les différents types d'entreprises de la nouvelle économie créées par des femmes, certaines données ont été colligées au tableau 8.

Il ressort que les femmes du groupe majoritaire français sont plus susceptibles de créer des entreprises fondées sur le savoir que les femmes des deux autres groupes, lesquels sont plus fortement concentrées dans les entreprises de services avec TI. Les différences entre ces deux derniers groupes ne sont pas significatives.

Tableau 8 : Type d'entreprises de la nouvelle économie créées par des femmes, selon l'origine immigrante à Montréal (2006)

\begin{tabular}{|l|l|l|l|l|}
\hline Type d'entreprises & Français & Anglais & Autres & Ensemble \\
\hline TI Manufacturière & $8,1 \%$ & $6,5 \%$ & $11,5 \%$ & $9,0 \%$ \\
\hline TI Services & $29,4 \%$ & $45,2 \%$ & $44,8 \%$ & $36,0 \%$ \\
\hline Fondée sur le savoir & $62,5 \%$ & $48,4 \%$ & $43,7 \%$ & $55,0 \%$ \\
\hline \hline Ensemble & $100,0 \%$ & $100,0 \%$ & $100,0 \%$ & $100,0 \%$ \\
\hline (N) & 160 & 31 & 87 & 278 \\
\hline
\end{tabular}

Source : Les répertoires d'entreprises Scott, 2007 


\section{CONCLUSION}

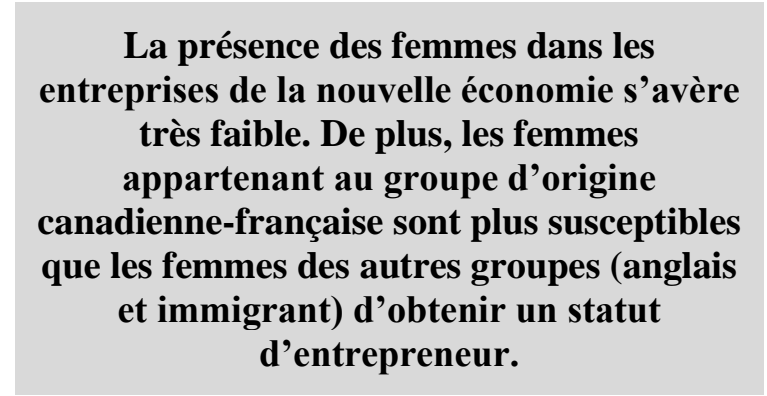

Cet article a réalisé une analyse comparative de la situation des femmes entrepreneures du groupe d'origine immigrante. L'attention a été centrée sur la présence des femmes ayant un poste de responsabilité dans l'entreprenariat d'affaires et sur l'impact différentiel que peut avoir le fait d'appartenir à un groupe d'origine immigrante. Les résultats obtenus à partir de l'analyse des données des répertoires Scott concernant la région du Montréal métropolitain sont riches et variés. Les entreprises de la nouvelle économie représentent environ $12 \%$ des affaires dans la région métropolitaine de Montréal, dont la majorité sont de l'économie du savoir. La présence des femmes dans les entreprises de la nouvelle économie s'avère très faible. De plus, les femmes appartenant au groupe d'origine canadienne-française sont plus susceptibles que les femmes des autres groupes (anglais et immigrant) d'obtenir un statut d'entrepreneur. Enfin, il n'y a pas d'écarts significatifs entre les femmes des trois origines différentes en termes du type d'entreprises qu'elles dirigent.

Nous avons aussi remarqué qu'en dépit des avantages comparés que détiennent les femmes d'origine française si on les compare à leurs équivalents masculins, il semble que le groupe d'origine française pris dans son ensemble est sous-représenté dans la structure entrepreneuriale de la région métropolitaine de Montréal. Par ailleurs, le groupe d'origine immigrante semble surreprésenté.

En bref, les résultats de cette étude sont intéressants. En effet, ils ont des implications en termes de question à approfondir telles que les difficultés d'accès des groupes aux fonds de démarrage et plus particulièrement des femmes entrepreneures d'origine immigrante et les facteurs de succès des entreprises dirigées par des femmes immigrantes.

\section{BIBLIOGRAPHIE}

${ }^{1}$ Paré, S. (2002). «Entrepreneurship ethnique au féminin à Montréal », Tremblay, D.-G. et Dagenais, L.-F. (dir.), Ruptures, segmentations et mutations du marché $d u$ travail, Québec, PUQ, 277-290. Paré, S. (2006). «La place des femmes immigrantes dans la nouvelle économie à Montréal : une exploration », Tremblay, D.-G. et Tremblay, R. (dir.), La compétitivité urbaine à l'ère de la nouvelle économie : enjeux et défis, 2006, Québec, PUQ, 353-368. Paré, S., Menzies, T. V., Filion, L. J. et Brenner, G. A. (2008). "Social capital and co-leadership in ethnic enterprises in Canada", Journal of Enterprising Communities: People and Places in the Global Economy, vol. $2, \mathrm{n}^{\circ} 1,52-72$.

${ }^{2}$ Polèse, M. et Shearmur, R. (2005). «Économie urbaine et régionale », Paris, Economica.

${ }^{4}$ Armstrong, P. (1996). «The Feminization of the Labour Force: Harmonizing down in a Global Economy », Bakker, I. (Ed.), Rethinking, restructuring: Gender and change in Canada, Toronto, University of Toronto Press, 29-54. Butler, J.S. et Kosmetsky, G. (2004). Immigrant and minority entrepreneurship: the continuous rebirth of American communities, Praeger Publishers, Wesport, CT, p. 200. McIsaac, E. (2003). «Immigrants in Canadian Cities: Census 2001-What do the Data Tell Us? » Policy Options, 58-63.

${ }^{5}$ Portes, A., Guarnizo, L. E. et Haller, W. J. (2002). «Transnational Entrepreneurs: An Alternative Form of Immigrant Economic Adaptation », American Sociological Review, vol. 67, $\mathrm{n}^{\circ} 2$, 278-298. Portes, A. et Bach, R. L. (1985). Latin journey: Cuban and Mexican immigrants in the United States. University of California Press, 387 p.

${ }^{6}$ Carr, D. (1996). «Two paths to self-employment? Women's and men's self-employment in the United States », Work and Occupations, vol. 23, $\mathrm{n}^{\circ}$ 1, 26-53. Loscocco, K.A., Robinson, J., Hall, R. A. et Allen, J. A. (1991). «Gender and small business success: an inquiry into women's relative disadvantage », Social Forces, vol. 70, $\mathrm{n}^{\circ} 1$, 65-85. Loscocco, K.A. et Robinson, J. (1991). "Barriers to women's smallbusiness success in the United States", Gender and Society, vol. 5, $\mathrm{n}^{\circ}$ 4, 511-532.

${ }^{7}$ Anna, A.L., Chandler, G. N., Jansen, E., Mero, N. P. (2000). Women business owners in traditional and non-traditional industries, Journal of Business Venturing $15: 279-303$ (p. 281). 
${ }^{8}$ Portes, A. et Rumbaut, R. G. (2006). Immigrant America: a portrait, University of California Press, $3^{\mathrm{e}}$ édition, $460 \mathrm{p}$.

${ }^{10}$ Julien, P.-A. et Morin, M. (1996). Mondialisation de l'économie et PME québécoises, Québec, PUQ, 204 p.

${ }^{11}$ Fontan, J.-M., Klein, J-L. Tremblay, D.-G. (2005). Innovation socio-territoriale et reconversion économique : Le cas de Montréal, France, L'Harmattan. ${ }^{12}$ Amabille, S. et Gadille, M. (2006). «Les usages d'Internet, facteurs de compétitivité de la "PME réseau" ? ", Finance Contrôle Stratégie, vol. 9, n 1 , 35-57. Julien, P.-A. (1997). Les PME: bilan et perspectives, Québec, PUQ. Kathuria, R. et Maheshkumar, J.P. (2006). «Environmental Influences on Corporate Entrepreneurship: Executive Perspectives on the Intemet », International Entrepreneurship and Management Journal 3(2), p. 127-144.

${ }^{13}$ KIRBY, D. et Cox, J. (2006). «Guest editorial: New Technology Based Firms in the Knowledge Economy », International Entrepreneurship and Management Journal 2, 139-144.

${ }^{14}$ Cadieux, L., Lorrain, J., Hugron, P. (2002). «La succession dans les entreprises familiales dirigées par les femmes : une problématique en quête de chercheurs ", Revue internationale PME 15(1):115-130.

${ }^{15}$ Paré, S. (2004). «Markers of Identity in Capital Markets: Ethnic Businesses and Female Entrepreneurs », Canadian Diversity, Intersections of Diversity, vol. 3 , n ${ }^{\circ}$ 1, p. 26-30.

${ }^{16}$ Fuller-Love, N., Lim, L., Akehurst, G. (2006). "Guest Editorial: Female and Ethnic Minority Entrepreneurship", International Entrepreneurship and Management Journal 2(4): 429-439.

${ }^{17}$ Candau, P. et R. Guir (1979). «Théorie de la séparation de la propriété et du contrôle et ethnicité, Revue d'économie industrielle 10(1): 53-65. 


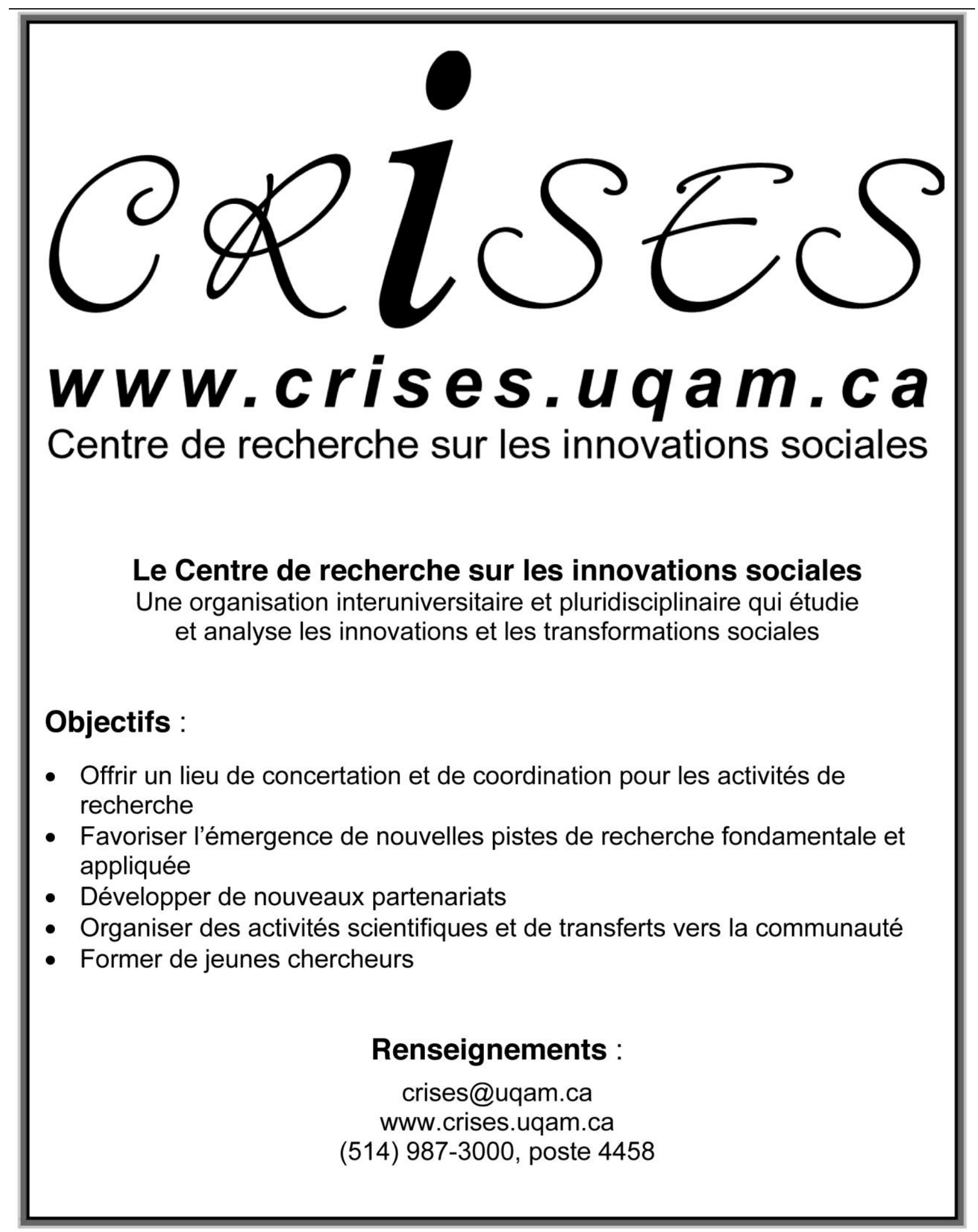

